Voix et Images

voixetimages

\title{
Quatre échos du divin moi
}

\section{André Brochu}

Volume 23, numéro 3 (69), printemps 1998

Le récit littéraire des années quatre-vingt et quatre-vingt-dix

URI : https://id.erudit.org/iderudit/201395ar

DOI : https://doi.org/10.7202/201395ar

Aller au sommaire du numéro

Éditeur(s)

Université du Québec à Montréal

\section{ISSN}

0318-9201 (imprimé)

1705-933X (numérique)

Découvrir la revue

\section{Citer cet article}

Brochu, A. (1998). Quatre échos du divin moi. Voix et Images, 23(3), 603-606. https://doi.org/10.7202/201395ar d'utilisation que vous pouvez consulter en ligne.

https://apropos.erudit.org/fr/usagers/politique-dutilisation/ 


\title{
Poésie
}

\section{Quatre échos du divin moi}

\author{
André Brochu, Université de Montréal
}

Très différents les uns des autres, les poètes dont je parlerai ont en commun une même passion du moi fait langage et ouvert, par là même, à la multiple vérité du monde.

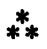

Serge Patrice Thibodeau est, sans contredit, la révélation des années quatre-vingt-dix en poésie québécoise ${ }^{1}$. Certains de ses recueils méritent de figurer aux côtés des plus grands de notre littérature. Je pense au Quatuor de l'errance suivi de La traversée du désert, et à Dans la cité suivi de Pacifica ${ }^{2}$. L'ampleur du propos, du rythme, la maîtrise du discours, la sûreté de la langue, la hauteur de l'inspiration, la nouveauté d'un langage poétique fondé sur l'enchaînement dynamique des idées plus que sur les images sémantiques (la métaphore, notamment), tout cela étonne et ravit. Thibodeau est extraordinairement libre de préjugés, et, grâce à lui, le poète redevient ce qu'il a pu être dans des textes majeurs de la littérature mondiale, mais très peu et même jamais dans la nôtre: un prophète, nourri de l'intuition profonde du divin, du sacré. Thibodeau est nourri aussi de solitude puisque son lieu d'élection, fréquenté patiemment et dangereusement par lui qui en a conservé la trace dans de grandioses poèmes, est le désert. Le désert, où parle la trans- cendance que la ville réduit au silence.

Serge Patrice Thibodeau a publié d'autres recueils qui se comparent avantageusement à la production courante, mais ne sont pas soutenus par les mêmes ambitions que les deux livres ci-dessus mentionnés. Tel est le cas de Nocturnes ${ }^{3}$. L'on y trouve des accents plus intimistes que ceux des grandes œuvres, et il en constitue de ce fait un précieux complément, mais le recueil, malgré ses dimensions appréciables, peut laisser le lecteur sur son appétit. D'ailleurs les premières sections, qui contiennent les textes les plus élaborés, sont nettement plus inspirées que les dernières. Les strophes régulières (constituées de vers libres) font un encadrement efficace pour un discours d'un âpre lyrisme, axé moins sur le sentiment que sur l'intensité, presque la violence, du désir. Thibodeau est un prophète et un mystique, mais non un elfe; et le désir du divin n'exclut nullement pour lui celui des corps. Des corps masculins, dont les attraits sont détaillés avec précision, et une liberté, une honnêteté qui enlèvent à ces évocations ce qu'elles pourraient avoir de choquant pour certains.

D’un ami, le poète écrit: "Il est l'action, il est l'urgence et le feu. . (p.49) Et c'est exactement les termes par lesquels on peut caractériser la poésie de Thibodeau. L'action, qui 
prend l'idée et la pousse à bout, la mène à la confrontation avec l'indicible. Le feu, qui est vie, sperme, désert, divin. Dans ce contexte, toutes métaphores sont dépassées:

Les rythmes règnent.

Je m'y soumets sans façons, sans dire adieu: (p. 44)

Au cours des années quatrevingt, Claude Beausoleil a publié plusieurs recueils considérables, d'un lyrisme éloquent et parfois prenant, qui exploitaient avec une généreuse inspiration, dans le cadre de longs poèmes, des formules ou matrices consistant en reprises d'éléments lexicaux ou formels. Depuis, il a expérimenté des factures plus brèves, comme dans Le grand bôtel des étrangers, récemment primé ${ }^{4}$. Quatre échos de l'obscur ${ }^{5}$ appartient à cette production plus sobre et plus courante puisqu'elle rappelle la plupart des parutions en poésie québécoise.

Toutefois, si l'on y regarde de près, les poèmes relativement courts de ce dernier recueil s'insèrent dans un programme très précis qui fait d'eux les engrenages d'une seule et même machine. Il y a quatre sections. Chacune d'elles fait écho c'est le cas de le dire - à un événement : la rupture amoureuse. Et, comme le suggère l'illustration de la page couverture, les échos sont de plus en plus sombres, douloureux. Nous sommes dans un univers à la fois de la répétition (de l'obsession) et de la progression.

La répétition se marque non seulement dans l'évocation du drame amoureux, mais dans la composition même. Chaque section compte quinze poèmes. Le premier comporte une strophe de cinq vers (le deuxième étant plus long et s'écrivant sur deux lignes), une autre de trois vers très brefs, et une dernière de quatre vers dont le dernier est plus long. Et il en va ainsi pour l'ensemble du recueil. Les deuxièmes poèmes de chaque section ont une première strophe de cinq à sept vers, une deuxième de huit, une dernière d'un seul, etc. Si on lit les poèmes en parallèle, on obtient, au premier vers du premier poème de chaque division, les énoncés suivants :

Un pareil destin nous traque, et nous le défions; (p. 9)

La langue est le seuil du temps, nous l'éprouvons; (p. 27)

Une image glisse du jour retourné d'ogives; (p. 45)

Le souffle est une syntaxe mimée, nous l'imprimons. (p. 63).

Les contenus sémantiques (destin, langue, image, souffle) sont très différents, les énoncés construits évoquent des réalités parfaitement hétérogènes (encore que le défi et l'épreuve, dans les deux premiers vers cités; la langue et l'image, dans les deuxième et troisième, puissent présenter quelque parenté de signification), mais la construction, à peu de détails près, est identique et voulue telle. On ne pourra pas dire que les poèmes manquent de rigueur, puisqu'ils se constituent en programme les uns pour les autres et assurent ainsi la constance d'une prosodie.

La structure étant donnée, on aimerait toutefois que le contenu ait lui aussi quelque rigueur, et c'est là que la poésie de Beausoleil peut s'avérer décevante. Le message est trop souvent - on pourrait dire : 
toujours - constitué de l'extérieur, à partir d'une idée, d'un programme. La nécessité interne est absente comme le montre bien un vers extravagant et fabriqué tel que le suivant: "Le souffle est une syntaxe mimée, nous l'imprimons." Seul leur caractère flou permet aux énoncés de s'inscrire, d'une part, dans un registre signifiant qui crée l'illusion de dire quelque chose, qui assure donc tant bien que mal la transitivité de l'écriture; et de répondre, d'autre part, aux exigences arbitraires du programme dans lequel ils puisent une insuffisante légitimité.

\section{**}

La vie est belle, aucun intellectuel n'empêchera Philippe Haeck de le penser. Dans Je ne sais pas ${ }^{6}$, il propose, en cent soixante-douze poèmes-essais qui sont autant d'essais-poèmes, une vision du monde digne d'Alexandre le bienheureux ${ }^{7}$ (la paresse en moins), et que, pour ma part, je trouve fort agréable, même si la poésie m'y semble souffrir quelque peu de la morale, des bonnes intentions, de cette naïveté, au fait pas si naïve, que l'auteur se plaît à cultiver.

En tout cas, cet optimisme vaut bien le pessimisme courant que Haeck a déjà reproché à l'un de nos plus grands poètes, et il permet des trouvailles. Car l'auteur ne se cantonne pas dans le créditisme ${ }^{8}$ intellectuel où il semble parfois verser. Aimer la vie, l'amour, le pauvre, le nu, le simple, l'ordinaire, l'épouse "compagne de toute une vie - (p. 41), les " yeux de vos enfants" (p..41), les élèves dont on est le maître souriant, dont on est l'élève même, ce n'est pas une perversion de l'esprit. C'est retrouver, peut-être, les vertus qui caractérisaient la société paysanne dont nous provenons, et refuser les artifices culturels, le * couinement général (p. 51), la "machine sociale " aliénante (p. 73). Travailler à être un "athlète de la joie " (p. 31), voilà une occupation qui dépasse l'individualisme, tout en l'assumant. Car c'est l'autre qui fonde le moi - «à nouveau les autres viennent en moi, je recommence à avoir un moi . (p. 14); l'intersubjectivité fait le sujet ${ }^{9}$.

Sur le plan formel, le livre est constitué de proses qui prennent tantôt allure de récits, tantôt de pensées, tantôt de fantaisies plus proprement lyriques. Ce qui assure l'unité de ces textes, c'est un ton particulier à l'auteur, auquel contribuent certains procédés comme l'absence de tout point d'interrogation (les questions sont assez fréquentes); la présence de mots agglutinés, avec trait d'union (idées-platras, musique-silence, homme-femme, pluie-vent...) ou non (propos chair offerte, propos tombeaux...), ou même fusionnés (coresprit, chairâme...). L'invention concerne davantage la dimension lexicale que la dimension thétorique. La recherche d'images neuves n'est pas une priorité de l'auteur, qui met plutôt l'accent sur la prospection des aspects du quotidien (banal) susceptibles de renouveler l'engagement de tout le corps et de tout l'esprit à promouvoir la joie, c'est-à-dire à assurer leur insertion dans la féconde magie de la vie. Cela produit-il de la poésie? Oui, si la poésie peut être énoncé de sagesse, donc expression d'une morale, d'une philosophie, et prise en compte de la vérité quotidienne. Sagesse, et non savoir: “ je ne 
sais pas", comme le dit bien le titre. Poésie de l'âme, quand l'âme est aussi sexe, tendresse, amour, ouverture à la jouissance-connaissance des autres et de soi. Seule manque sans doute, chez Haeck, cette aventure spécifique du langage qui déporte le message vers des registres inconnus, où le sens s'auto-invente sous. la pression des mots. L'aventure est pourtant présente au premier degré, dans la représentation courageuse des vertus familières.

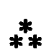

Roland Marquis publie, à quarante-sept ans, son premier recueil de poèmes. Jusqu'ici, il n'avait fait paraître qu'un recueil de nouvelles (en 1992). Une manifestation aussi tardive pourrait être le signe d'une vocation peu assurée. Ce n'est nullement le cas. Le miroir des espèces ${ }^{10}$ est un fort beau livre, qui révèle une voix parfaitement maîtrisée et originale. De façon évidemment approximative, on pourrait la situer quelque part entre celles d'Anne Hébert et de Lautréamont.

L'univers moral, affectif, physique que dépeint Marquis est merveilleusement étrange, comme s'il relevait d'une grammaire ethnologique juste assez familière pour accrocher notre intérêt, mais propre à déconcerter le plus aguerri des esprits. Les rapports entre les êtres sont esquissés en quelques mots seulement, très simples et absolument énigmatiques. Une des lois de cet univers est sans doute l'égalité de statut entre l'espèce humaine et les espèces animales, toutes réglées par la cruauté. De là le titre: les espèces, qui se valent et qui se reflètent mutuellement, font du monde un spectacle où la passion revêt les formes les moins usitées (deux hommes s'aiment, la fille aime son père, la reine son bourreau, l'infirmière son malade...). Et ce spectacle existe, en fin de compte, pour " l'œil unique " au fond duquel tout tombe (p. 67), et qui est l'avatar du ventre des origines.

Dans l'hermétisme du recueil, il y a la trace d'une difficulté de l'auteur à communiquer, manifeste aussi dans la rareté de ses publications. Souhaitons que l'œuvre se fasse plus abondante et que son obscurité, si fascinante soit-elle, s'atténue un peu au profit du riche déploiement de l'imaginaire qu'on est en droit d'attendre d'elle.

1. Est-il besoin de préciser que Serge Patrice Thibodeau, né en Acadie, dans la vallée de la Madawaska, est aussi québécois que l'était Gabrielle Roy, née au Manitoba?

2. Les deux recueils ont paru aux éditions de l'Hexagone, en 1995 et en 1997.

3. Serge Patrice Thibodeau, Nocturnes, Trois-Rivières, Écrits des Forges, 1997, $96 \mathrm{p}$.

4. Claude Beausoleil, Le grand hôtel des étrangers, Trois-Rivières/Luxembourg, Écrits des Forges/Éditions Phi, 1997. Prix Alain-Grandbois de l'Académie des Lettres du Québec, et Grand Prix du Festival international de poésie de Trois-Rivières.

5. Id., Quatre échos de l'obscur, Trois-Rivières, Êcrits des Forges, 1997, 81 p.

6. Philippe Haeck, Je ne sais pas, Montréal, VLB éditeur, 1997, $158 \mathrm{p}$.

7. Film culte d'Yves Robert (1967), avec Philippe Noiret.

8. Mouvement politique des années soixante, dirigé par Réal Caouette au fédéral et Camille Samson au provincial, pour représenter les classes défavorisées. On y dénonçait les * requins de la finance. et les intellectuels.

9. Thème développé par Alain Renaut dans L'ère de l'Individu, Paris, Gallimard, coll. - Bibliothèque des idées ", 1989, 302 p.

10. Roland Marquis, Le miroir des espèces, Montréal, Les Herbes rouges, 1997, 76 p. 